\title{
Are We Aware of the Danger?
}

\begin{abstract}
Usage of energy drinks has been increasing in our country in recent years as it has been in the whole world. We aimed to present ingredients and side effects of energy drinks which are consumed mostly by adolescents and young adults. Additionally, we aimed to discuss recent literature related to energy drinks which are thought to serve as a step in the way of alcohol consumption and addiction in recent years.
\end{abstract}

Keywords: Energy drinks; Ingredients of energy drinks; Adolescent; Side effects; Risks

\section{Are We Aware of the Danger?}

Consumption of energy drinks has a tendency to increase in our country in the last ten years as it has in the whole world. Marketing, usage and popularity of these beverages have been increased by some popular approaches such as these products increase energy of someone, produce sleeplessness, decrease tiredness especially during driving and studying, and enhance reaction time [1]. We tried to look at scientific approaches to those products which have a wide usage in popular culture.

Energy drinks introduced to market first in Austria in 1987, and they entered the market in the USA in 1997. The intended population of these products is the age group between 11 to 35 years. It is known that usage of energy drinks causes some social negative effects for example; increasing the risk of getting into a brawl, and usage of addictive drugs especially marihuana and alcohol addiction and smoking [2]. It has been noted that emergency admissions related to consumption of energy drinks doubled in the USA in the last 4 years [3].

\section{Which ingredients are Present in Energy Drinks?}

Although energy drinks may contain different ingredients as the producer changes caffeine is present in all of them, and they include different amounts of taurine, glucuronolactone, guarana, B complex vitamins, ginseng/guarana extracts, ginkgo biloba, carnitine, carbohydrates containing fructose, and sucrose, and various sweetening aromas [2].

\section{Caffeine}

Caffeine is an antagonist of adenosine receptors, and it has a stimulant effect on neuronal pathways in both central and peripheral nervous systems. The most important stimulant effect of energy drinks is caused by caffeine. As a result of its ergogenic effect caffeine increases heart rate, blood pressure and respiratory rate. Because that condition is accepted as a state of relatively enhanced endurance caffeine has become a cheaper, legal, and easily accessible substance. All of those factors may be listed in reasons why caffeine-containing beverages are used more commonly by adolescents and young individuals. Ninetynine $\%$ of orally taken caffeine is absorbed by gastro-intestinal system, and passes blood brain barrier and placental barrier easily. It reaches peak plasma concentration in 50 to 75 minutes. It augments release of noradrenaline. Effects on cardiovascular

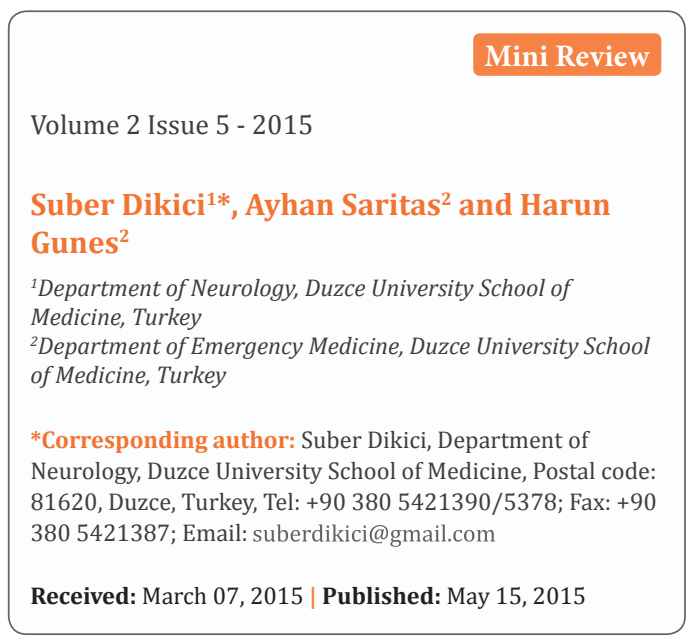

system become more apparent after stimulation of dopamine receptors. Supraventricular and ventricular tachy-arrhythmias may occur after caffeine over-dose [4]. Caffeine leads to some side effects such as peripheral vasodilatation, restlessness, anxiety, tremor and changes of mental status by directly affecting respiratory system and vasomotor centers of brain [5].

Oral caffeine intake of adults and children (12 years of age or older) should be restricted to 100 to 200 milligrams not more often than every 3 to 4 hours. Amount of caffeine in energy drinks differs between 80 to $300 \mathrm{mg}$. But caffeine intake increases as number of portions taken in a day increases. In addition, some ingredients like guarana include occult caffeine thus amount of caffeine taken reaches considerably larger amounts as compared to amount depicted on product label [6]. Moderate amounts of caffeine like 85-250 mg enhance alertness, alleviate tiredness, and improve concentration. At larger doses like 250-500 mg; restlessness, hyperactivity, nervousness, insomnia, tremor and gastro-intestinal complaints may occur. Muscle spasms, myocardial irritability, arrhythmias and epileptic seizures may be seen when 15-30 mg of caffeine is taken [7].

Additionally, caffeine may lead to water loss and dehydration by causing a diuretic effect. It is suggested that dehydration secondary to use of energy drinks is related to caffeine.

\section{Guarana}

Guarana is a plant growing in Brazil and Venezuela. It is included in various amounts in different energy drinks changing according to manufacturer. Three to five gr of Guarana contains approximately $250 \mathrm{mg}$ caffeine, thus increases the caffeine content of energy drinks [8].

\section{Taurine}

It is a natural amino-acid found in foods like meat and fish. A normal diet includes 20-200 mg of Taurine. The amount of taurine in energy drinks changes between 600 to $1000 \mathrm{mg}$. Although there is no reported side effect of taurine alone, it is suggested to be responsible for athlete deaths in Europe when taken together with caffeine [6]. 


\section{Ginseng}

It is a stimulating herbal agent derived from roots of the plant "Panax ginseng", and have been used since ancient times in the Far East. It is used due to its effects such as improving immune functions, enhancing physical endurance, decreasing stress and increasing libido [9]. Positive effects of ginseng on memory, concentration and cognitive functions lacks scientific evidence in contrast to energy drink manufacturers' claims [6]. Side effects like insomnia, stomach pain, edema, headache, vertigo, euphoria and mania may be seen in common use [8].

\section{Yohimbine}

It is a strong herbal stimulant, and used in the treatment of erectile dysfunction due to its aphrodisiac effect. Additionally, it is used in the treatment of sexual side effects of anti-depressants [10]. It has serious side effects such as anxiety, insomnia, headache, vertigo, skin flushing, panic attack, hallucinations, increase in heart rate and high blood pressure. It may cause epileptic seizures and renal failure. It is an agent which should not be used by people a renal, cardiac or hepatic disease or psychiatric disorders [11].

\section{Bitter orange}

It is an herbal agent found in some trade products. It has a therapeutic effect when used alone up to $200 \mathrm{mg}$ /day but they cause an addictive stimulant effect when taken together with caffeine, and guarana as in the case of energy drink intake [6]. Additionally, it is suggested that the addictive stimulant effect makes risk of cardiovascular effects to appear. It is important to state that bitter orange has an increasing effect on heart rate and blood pressure which contributes to the appearance of cardiovascular risks [6].

\section{Effects of Energy Drinks}

It is known that the primary risky substance energy drinks contain is caffeine, and the other substances in energy drinks potentiating effects of caffeine increase the risk. High doses of caffeine may cause palpitations, hypertension, diuresis, central nervous system stimulation, nausea, vomiting, metabolic acidosis, epilepsy and cerebral ischemia [12,13]. Usage of energy drinks in the young makes them to exhibit unfavorable behaviors, and forms the first step in the way to start smoking or usage of the other addictive agents [5]. Although use of energy drinks and alcohol simultaneously must be avoided is known that they mostly used together. It has been claimed that drinking energy drinks alone is an independent risk factor for alcohol addiction even if any alcohol containing drinks are not taken simultaneously [14]. It is especially important to note that perception of energy drinks which actually increase the risk of drug addiction as innocent is a serious public health problem.

High calorie content of energy drinks exceeds daily calorie requirements, and leads to obesity [6]. It makes the subject of energy drinks more important to know that energy drinks and the other related beverages contribute to increasing childhood obesity. Increased caffeine intake results in serious side effects like hypertension, insomnia, chronic headaches, motor tics, irritability and learning difficulties [8]. It was reported that usage of sweetened beverages and sodas is related to stroke occurrence
[15]. However sweetened beverages and sodas are not included in the group of energy drinks because their ingredients and amounts of ingredients differ from those of energy drinks [16]. Common central nervous system side effects of energy drinks include headache, anxiety, agitation, tremor, confusion, changes in mental status, new-onset epilepsy, cerebral ischemia and transient ischemic attack $[6,8,13,17]$.

Number of the energy drink-related deaths as a result of chest pain, increased heart rate, hypertension, ventricular tachycardia, atrial fibrillation, arrhythmia and myocardial infarction has a tendency to increase. Intake of energy drinks after mixing them with alcohol makes side effects especially the ones related to cardiovascular system to be more common and more serious [18]. Side effects of energy drinks related to large amounts of caffeine content such as nausea, vomiting, abdominal pain, diarrhea, anorexia, gastro-esophageal reflux and insufficiency in intestinal movements cause life quality of one to decrease dramatically [19]. Energy drinks are extensively marketed by claiming that they improve performance, increase endurance, enhance attention, and help losing weight which does not have scientific evidence. Masculine risk taking messages originating from advertising complements and presentation of energy drinks as a legal alternative to cocaine have a role in increasing use of those products. It is a reality that all of those factors lead the condition to reach a dead end [20].

Marketing of energy drinks was banned in France in 2008 due to concerns about their side effects but was allowed again later. Although there are no limitations to marketing of energy drinks in the European Union (EU) some limitations are present in some countries of the EU. For example, they are not allowed to be sold to someone under the age of 15 , and are sold in pharmacies only in Sweden [21]. Unfortunately, there are no limitations to marketing of energy drinks and advertisements of those products are commonly shown on television in "the prime time" in our country. Some efforts to limit caffeine content of energy drinks due to its possible side effects are present in America and Europe where it is very well known that energy drinks are not innocent [21].

\section{Conclusion}

We suggest that it is necessary to raise public's awareness of improper use of energy drinks which have a potential to threat public health. Legal limitations to sale of energy drinks to children and adolescents as in the case of cigarettes and alcohol, studies about the side effects of those products, and sharing the scientific data on that subject may be helpful.

\section{References}

1. Attila S, Cakir B (2011) Energy-drink consumption in college students and associated factors. Nutrition 27(3): 316-322.

2. Miller KE (2008) Energy drinks, race, and problem behaviors among college students. J Adolesc Health 43(5): 490-497.

3. The DAWN Report (2013) Update on emergency department visits involving energy drinks: a continuing public health concern. Substance Abuse Services and Mental Health Administration.

4. McCarthy DM, Mycyk MB, DesLauriers CA (2008) Hospitalization for caffeine abuse is associated with abuse of other pharmaceutical products. Am J Emerg Med 26(7): 799-802. 
5. Luebbe AM, Bell DJ (2009) Mountain Dew or mountain don't?: a pilot investigation of caffeine use parameters and relations to depression and anxiety symptoms in 5th- and 10th-grade students. J Sch Health 79(8): 380-387.

6. Clauson KA, Shields KM, McQueen CE, Persad N (2003) Safety issues associated with commercially available energy drinks. J Am Pharm Assoc 48(3): e55-e63.

7. Iyadurai SJ, Chung SS (2007) New-onset seizures in adults: possible association with consumption of popular energy drinks. Epilepsy Behav 10(3): 504-508.

8. Babu KM, Church RJ, Lewander W (2008) Energy drinks: The new eye opener for adolescents. Clin Pediatr Emerg Med 9(1): 35-42.

9. Hong B, Ji YH, Hong JH, Nam KY, Ahn TY (2002) A double blind crossover study evaluating the efficacy of Korean red ginseng in patients with erectile dysfunction: a preliminary report. J Urol 168(5): 2070-2073.

10. Wehr TA (1990) Manipulations of sleep and phototherapy: nonpharmacological alternatives in the treatment of depression. Clin Neuropharmacol 13(Suppl 1): S54-S65.

11. Kearney T, Tu N, Haller C (2010) Adverse drug events associated with yohimbine-containing products: a retrospective review of the California Poison Control System reported cases. Ann Pharmacother 44(6): 1022-1029.

12. Brown IJ, Stamler J, Van Horn L, Robertson CE, Chan Q, et al. (2011) Sugar-sweetened beverage, sugar intake of individuals, and their blood pressure: international study of macro/micronutrients and blood pressure. Hypertension 57(4): 695-701.
13. Dikici S, Saritas A, Besir FH, Tasci AH, Kandis H (2013) Do energy drinks cause epileptic seizure and ischemic stroke? Am J Emerg Med 31(1): 274.e1-e4.

14. Snipes DJ, Jeffers AJ, Green BA, Benotsch EG (2015) Alcohol mixed with energy drinks are robustly associated with patterns of problematic alcohol consumption among young adult college students. Addict Behav 41: 136-141.

15. Bernstein AM, de Koning L, Flint AJ, Rexrode KM, Willett WC (2012) Soda consumption and the risk of stroke in men and women. Am J Clin Nutr 95(5): 1190-1199.

16. Saritas A, Dikici S, Gunes H (2015) Adverse effects of energy drinks. Am J Emerg Med 33(3): 461-462.

17. Dikici S, Saritas A, Kilinc S, Guneysu S, Gunes H (2015) Does an energy drink cause a transient ischemic attack? Am J Emerg Med 33(1): 129. e5-e6.

18. Dikici S, Aydın LY, Kutlucan A, Ercan N (2012) What do we know about energy drinks?. Dicle Medical Journal 39(4): 609-613.

19. Rath M (2012) Energy drinks: what is all the hype? The dangers of energy drink consumption. J Am Acad Nurse Pract 24(2): 70-76.

20. Malinauskas BM, Aeby VG, Overton RF, Carpenter-Aeby T, BarberHeidal K (2007) A survey of energy drink consumption patterns among college students. Nutr J 6: 35.

21. Bundesinstitut für Risikobewertung (2008) New human data on the assessment of energy drinks. Federal Institute for Risk Assessment. 